OPEN ACCESS

Edited by: Andrew Steele, Bill and Melinda Gates Foundation, United States

Reviewed by: Owen Kavanagh, York St John University,

United Kingdom Rupsa Basu,

Technovax Inc, United States

*Correspondence: Nazish Bostan nazishbostan@comsats.edu.pk

Specialty section: This article was submitted to Vaccines and Molecular Therapeutics, a section of the journal Frontiers in Immunology

Received: 14 May 2020 Accepted: 21 September 2020 Published: 02 October 2020

Citation:

Sadiq A and Bostan N (2020) Comparative Analysis of G1P[8] Rotaviruses Identified Prior to Vaccine Implementation in Pakistan With Rotarix ${ }^{\mathrm{TM}}$ and RotaTeq ${ }^{\mathrm{TM}}$ Vaccine Strains.

Front. Immunol. 11:562282. doi: 10.3389/fimmu.2020.562282

\section{Comparative Analysis of G1P[8] Rotaviruses Identified Prior to Vaccine Implementation in Pakistan With Rotarix $^{\mathrm{TM}}$ and RotaTeq ${ }^{\mathrm{TM}}$ Vaccine Strains}

\author{
Asma Sadiq and Nazish Bostan * \\ Department of Biosciences, COMSATS University (CUI), Islamabad, Pakistan
}

Group A rotavirus (RVA) is the leading cause of severe childhood diarrhea globally, even with all effective interventions, particularly in developing countries. Among the diverse genotypes of RVA, G1P[8] is a common genotype that has continued to pervade around the world, including Pakistan. Two universally accepted rotavirus vaccines-Rotarix ${ }^{\mathrm{TM}}$ and RotaTeq $^{\mathrm{TM}}$ contain the genotype G1P[8]. The current work was aimed at identifying differences between antigenic epitopes of Pakistan's G1P[8] strains and those of the two licensed vaccines. We sequenced $6 \mathrm{G} 1 \mathrm{P}[8]$ rotavirus strains previously reported in Rawalpindi, Islamabad, Pakistan in 2015 and 2016 for their outer capsid genes (VP7 and VP4). Phylogenetic analysis was then conducted in order to classify their specific lineages and to detect their association with strains isolated throughout world. Compared with the Rotarix ${ }^{\mathrm{TM}}$ and RotaTeq ${ }^{\mathrm{TM}}$ vaccine strains (G1-lineage II, P[8]-lineage III), our study G1-lineage I, P[8]-lineage IV strains showed 3 and 5 variations in the VP7 epitopes, respectively, and 13 and 11 variations in the VP4 epitopes, respectively. The G1 lineage II strains showed no single amino acid change compared to Rotarix ${ }^{\mathrm{TM}}$ (lineage II), but exhibited changes at 2 positions compared to RotaTeq ${ }^{\mathrm{TM}}$ (lineage III). So, this has been proposed that these G1 strains exist in our natural setting, or that they may have been introduced in Pakistan from other countries of the world. The distinct P[8]-lineage IV (OP354-like) strains showed twelve and thirteen amino acid variations, with Rotarix ${ }^{\mathrm{TM}}$ and RotaTeq $^{\mathrm{TM}}$ (lineages II and III) strains, respectively. Such findings have shown that the VP4-P[8] component of the G1P[8] strains circulating in Pakistan differs considerably from that of the vaccine viruses compared to that of the VP7-G1. To monitor the long-term effects of vaccines on the emergence of G1P[8] strains with different lineages, routine and successful monitoring of these strains will be crucial.

Keywords: G1P[8], rotavirus, genotype, antigenic epitopes, emergence 


\section{INTRODUCTION}

Rotavirus-related gastroenteritis (RVGE) is a significant global health concern contributing to 128,500 deaths among children younger than 5 years worldwide in 2016, as of the most recent data (1). While the rate of diarrhea-associated mortality has decreased globally over the last decade, the burden of rotavirus diarrhea remains significant in low-income settings (2). According to estimates from the WHO, approximately half of all rotavirus-associated deaths occurred in India, Nigeria, Pakistan and the Democratic Republic of Congo in 2013 (3). For an estimated 393,959 deaths under five years of age in children in Pakistan, 14,700 are due to rotavirus diarrhea (4). Pakistan is in its early stages of using rotavirus vaccines to combat childhood rotavirus infections (5).

Rotaviruses in the family Reoviridae are icosahedral and triple layered viruses with eleven segmented double- stranded RNA (ds-RNA) genome $(6,7)$. All eleven segments express six structural (VP1-VP4, VP6 and VP7) and six non-structural proteins (NSP1-NSP6) (8). Based on genes coding for the two outer capsid proteins (VP7 and VP4), RVAs are categorized into G (glycoprotein) and P (protease-sensitive) genotypes. Till now, 36 genotypes of $\mathrm{G}$ and $51 \mathrm{P}$ have been reported worldwide (9). The epidemiological studies indicate that at least six human RVA genotypes G1P[8], G2P[4], G3P[8], G4P[8], G9P [8], and G12P [8] are globally prevalent in human infections (10).

Rotavirus is a segmented virus that is continuously evolving (11). There is substantial variation in the distribution of rotavirus genotypes globally. The most prevalent strains that cause serious disease change year after year within and between country to country. The error-prone RNA-dependent polymerase, without proofreading ability, offers various evolutionary mechanisms for rotavirus, involving point mutation, reassortment, recombination, and interspecies transmission $(12,13)$.

The battle against rotavirus infection has been a big concern for WHO (World Health Organization) for many years (14). Four WHO pre-qualified vaccines are now eligible for global use and for procurement by Gavi, the Vaccine Alliance and UNICEF. These are the human monovalent (RV1) Rotarix ${ }^{\mathrm{TM}}$ (GlaxoSmithKline); human pentavalent (RV5) RotaTeq ${ }^{\mathrm{TM}}$ (Merck and Co., Inc); ROTAVAC ${ }^{\circledR}$ (Bharat Biotech); and ROTASIIL $^{\circledR}$ (Serum Institute of India) $(15-17)$. RotaTeq ${ }^{\text {TM }}$ include human VP7 (G1-G4) and VP4 (P[8]) genotypes, Rotarix $^{\mathrm{TM}}$ consists of a single human G1P[8] strain, whereas, Rotavac ${ }^{\circledR}$ is a monovalent human-bovine RV vaccine containing genotype (G9P[11]) and RotaSIIL ${ }^{\circledR}$ is a live attenuated humanbovine reassortant pentavalent RV vaccine containing VP7 genotypes (G1, G2,G3, G4, and G9) and VP4 genotype (P[5]) of bovine origin $(17,18)$.

In 2009, WHO recommended that all countries, and especially those with high child mortality rates for diarrhea, should implement rotavirus vaccines into their local immunization plans (19). By April 2020, 107 countries, of which 46 are eligible for Gavi, the Vaccine Alliance, have included rotavirus vaccines in their national or regional vaccination plans (20). The effectiveness of rotavirus vaccines is higher in developed, high-income countries (HICs) than in low income countries (LICs) and lower middle income countries (LMICs) $(19,21)$. Complicating factors such as nutritional status, age at infection, size of inoculum of infectious agent and interference by other enteropathogens may also impact on vaccine success in developing countries which suffer the greatest burden of rotavirus disease (22). Two additional rotavirus vaccines are available regionally, i.e., Rotavin-M1 in Vietnam and Lanzhou Lamb Rotavirus (LLR) in China $(23,24)$.

As rotavirus is continually altering its genetic makeup, it is therefore imperative to study how immunity derived from vaccines influences the development and spread of the vaccinetargeted major RVA strains (25). Vaccines that fail to eliminate a viral pathogen can apply pressures that cause changes in a microbial population's genetic composition (26). Close human animal encounters and rise in global migration can transmit new virus virulence factors or mutants into unaffected populations, giving these viruses an opportunity to gain biological competitiveness against the common wild-type strains existing in the population (11). Such variations can be detrimental to the efficacy of the vaccine. Although immune responses to vaccines may be affected by several factors, human genetic differences are thought to have a significant effect on vaccine response variability (27). In defined virus epitopes, positive selection of single amino acid mutations gradually generates diversity of the viruses (28). The vaccine virus will not trigger an immune response, identifies and eliminates the newly emerged virus. Thus ELISA or other neutralization studies performed for variant strains can be affected.

The Government of Pakistan, with support from GAVI, the Vaccine Alliance, included Rotarix ${ }^{\mathrm{TM}}$ vaccine in the EPI schedule in 2018 (7). The post-vaccination rotavirus studies regarding the effectiveness of RVA vaccines and their effects on the particular genotypes of the country have yet to be identified (29). Over the pre-vaccine age, surveillance data from Pakistan have shown that G1P [8] is one of the most common RVA genotypes circulating in Pakistan over the last decade $(30,31)$. G1P[8] strains are responsible for the majority of human RVA infections (32). The main objective of this study was therefore to investigate the disparity between the circulating wild G1P[8] strains collected prior to vaccine implementation in Pakistan with the Rotarix ${ }^{\mathrm{TM}}$ and RotaTeq ${ }^{\mathrm{TM}}$ strains. The findings of this study will lead to the potential establishment of the pre-vaccination background for comparison studies after vaccination.

\section{MATERIAL AND METHOD}

\section{Study Samples}

In the current study we selected six representative RVA G1P[8] strains based on the partial sequencing, phylogenetic analysis and their distinct circulating lineages. These specimens were already genotyped by partial sequencing in a hospital-based RVA surveillance study performed between January 2015 and December 2016 (7). The research population included children $<10$ years of age admitted with acute gastroenteritis in two hospitals in Rawalpindi, Islamabad, Pakistan, PIMS (Pakistan Institute of Medical Sciences and BBH (Benazir Bhutto 
Hospital). The complete sequences for six G1P[8] strains that were obtained in this study were needed for further comparative vaccine analysis.

\section{RNA Extraction}

Fecal suspensions (10\%) were prepared by adding $100 \mathrm{mg}$ of fecal sample in $1 \mathrm{ml}$ of phosphate buffer saline (PBS) in a sterile eppendorf tube. In compliance with the manufacturer's instructions, a QIAamp viral RNA minikit (Qiagen) was used to extract viral RNA from the diluted stool content.

\section{RT-PCR and Sequencing}

Extracted RNA were denatured at $95^{\circ} \mathrm{C}$ for $2 \mathrm{~min}$, and RT-PCR was carried out using a Qiagen one-Step RT-PCR kit $\left(20 \mu \mathrm{l}\right.$ of $\mathrm{H}_{2} \mathrm{O}, 5 \mu \mathrm{l}$ of Qiagen One step RT-PCR buffer, $5 \mu \mathrm{l}$ of diluted RNA, $1.5 \mu \mathrm{l}$ of forward primer $(10 \mu \mathrm{M}), 1.5 \mu \mathrm{l}$ of reverse primer $(10 \mu \mathrm{M})$ and $1 \mu \mathrm{l}$ of RT-PCR enzyme mix. The full VP7 and VP4 genes (regions VP8* and $\mathrm{VP} 5^{\star}$ ) were amplified using the previously described primers $(25,33-35)$. RT-PCR was carried out with an initial RT step at $50^{\circ} \mathrm{C}$ for $30 \mathrm{~min}$; Taq polymerase activation was carried out at $95^{\circ} \mathrm{C}$ for $15 \mathrm{~min}$, followed by 40 cycles of amplification (denaturation $45 \mathrm{~s}$ at $94^{\circ} \mathrm{C}$, annealing at $45^{\circ} \mathrm{C}$ for $45 \mathrm{~s}$ for VP 4 and at $50^{\circ} \mathrm{C}$ for $45 \mathrm{~s}$ for VP7, extension $1 \mathrm{~min}$ at $72^{\circ} \mathrm{C}$ and final extension at $72^{\circ} \mathrm{C}$ for $10 \mathrm{~min}$ ). The PCR product was purified using the ExoSAP-IT ${ }^{\mathrm{TM}}$ clean-up kit (Thermofisher Scientific, USA) and sequenced using the BigDye Cycle Sequencing Kit (Applied Biosystems, USA). The sequencing was carried out using forward and reverse primers that were used for the RT-PCR. Following the initial sequencing reaction, an ethanol precipitation was carried out and the final product was loaded in the automated sequencer ABI PRISM 3130 (Applied Biosystems, USA). Furthermore, the primer-walking sequencing was conducted to cover the full sequence of segments VP7 and VP4 (36).

\section{Sequence and Phylogenetic Analysis}

Sequencing files were processed using Chromas v2.6.5 (Technelysium, Australia) and multiple sequence alignments were performed via CLUSTALW in the MEGA 6.0 (37). The maximum likelihood trees were built using the Kimura-2parameter model in MEGA 6. The amino acids and nucleotides differences were determined using P-distance model in MEGA 6.0. The structural analysis of VP7 (PBD 3FMG) and VP8 (PDB 1KQR) was carried out using UCSF Chimera-Molecular Modeling tool (38).

\section{Research Data Availability}

The data sets created for this analysis are available with following accession numbers on GenBank: (VP7) MT381737-MT381742 and (VP4) MT381743-MT381748.

\section{RESULTS}

\section{Phylogenetic and Sequence Analysis}

Phylogenetic trees were constructed on the basis of full-length sequencing of six Pakistani G1P[8] strains for the VP7 and VP4 gene segments. G1 component of our study strains clustered in two distinct lineages I and II on phylogenetic observation, while their counterpart $\mathrm{P}[8]$ clustered into lineages III and IV, respectively. Rotavirus strains used in the synthesis of Rotarix $^{\mathrm{TM}}$ and RotaTeq ${ }^{\mathrm{TM}}$ belong to lineages II and III, respectively. We analyzed the difference between nucleotide and amino acid (a) in antigenic regions for our G1P[8] strains and two globally available vaccines (Rotarix ${ }^{\mathrm{TM}}$, RotaTeq $^{\mathrm{TM}}$ ).

Our study VP7 lineage II strains (PAK41, PAK65 and PAK77) showed nucleotide and amino acid identities of $96.7 \%$ and $96.3 \%$, respectively to VP7 protein of Rotarix ${ }^{\mathrm{TM}}$ vaccine belong to lineage II. While, G1 lineage I strains (PAK88, PAK540 and PAK601) showed 93.2\% nucleotide and 94.2\% amino acid identities to that of Rotarix ${ }^{\mathrm{TM}}$ VP7 protein. On phylogenetic analysis these strains clustered closely with strain isolated from Iran (21/Iran), previously reported strains (PAK42 and NIH$\mathrm{BBH}$ 4698)) from Pakistan and strains isolated from all around the world in VP7-G1 lineage II. Our study VP7 lineage I strains exhibited nucleotide and amino acid identities of $91.2 \%$ and $93.5 \%$, respectively to VP7 protein of RotaTeq ${ }^{\mathrm{TM}}$ vaccine and showed $90.5 \%$ and $93.2 \%$ of nucleotide and amino acid identities, respectively to that of RotaTeq ${ }^{\mathrm{TM}} \mathrm{VP7}$ protein. On phylogenetic tree these strains showed close similarity with Indian G1 strains (IDH_73, DIB/RMRC-11-03-107, and DIB/RMRC-11-03-115), previously reported Pakistani strain (NIH-KGH-6045) and other worldwide reported strains in VP7-G1 lineage I (Figures 1, 2).

VP4 of our study P[8] RVA strains that grouped into lineage IV were more closely related on amino acid and nucleotide level (95.4\% and $91.7 \%$, respectively) to VP4 protein of RotaTeq ${ }^{\mathrm{TM}}$ (W-179-4-lineage II) than lineage III of RotaTeq ${ }^{\mathrm{TM}}$ (W-179-9). Phylogenetic analysis have revealed that these strains clustered closely with strains isolated from, Africa, China, Belgium Russia (Nov09-D187, E1545, and BE1400) and with strains isolated from other countries of the world inVP4-P[8] lineage IV. VP4 of Pakistani P[8] RVA strains clustering in lineage III showed nucleotide and amino acid identities (87.6\% and $92.3 \%$, respectively) to VP4 protein of Rotarix ${ }^{\mathrm{TM}}$ (A41CB052A) and ( $86.9 \%$ and $92.1 \%$, respectively) to the VP4 protein of RotaTeq ${ }^{\mathrm{TM}}$ (W179-4). Phylogenetic study have revealed that these strains grouped together closely with strains reported in Japan Indonesia (Tokyo-17-21 and STM387) and other worldwide strains in VP4-P[8] lineage III (Figures 1-3).

\section{Comparative Analysis of VP7-G1 and VP4- $P$ [8] Proteins of RVA Strains Circulating in Pakistan and Rotavirus Vaccines}

In the current study, we have compared the VP7 and VP4 (VP8* and $\mathrm{VP}^{\star}$ ) antigenic epitopes of 6 Pakistani G1P[8] rotavirus strains to those of the RVA vaccine (RotaTeq ${ }^{\mathrm{TM}}$ and Rotarix ${ }^{\mathrm{TM}}$ ) strains. The VP7 protein covers three vital antigenic regions (71a, 7-1b, 7-2). These regions contain 29 amino acid residues from position 87-291, based on strains found in rhesus and submitted with accession number AF295303 in GenBank (39).

When our study G1 strains (lineage I and lineage II) were compared to two vaccines (RotaTeq ${ }^{\mathrm{TM}}$ and Rotarix ${ }^{\mathrm{TM}}$ ), the region $7-1 b$ was highly conserved for both vaccines but differences were 


\begin{tabular}{|c|c|c|c|c|c|c|c|c|c|c|}
\hline \multicolumn{11}{|c|}{ Nucleotide Identities (\%) } \\
\hline & Lineage & Lineage & Rotari $^{\mathrm{TM}}$ & RotaTeq ${ }^{\text {TM }}$ & Rotarix $^{\mathrm{TM}}$ & Rota Teq ${ }^{\mathrm{TM}}$ & G1 of Rotarix ${ }^{\mathrm{TM}}$ & G1 of RotaT eq ${ }^{\mathrm{TM}}$ & $\mathrm{P}[8]$ of Rotarix ${ }^{\mathrm{TM}}$ & P[8] of RotaTeq ${ }^{\mathrm{TM}}$ \\
\hline $\begin{array}{l}\text { Strain } \\
\text { Name }\end{array}$ & VP7-G1 & VP4-P[8] & Limeage & Lineage & Lineage & Lineage & (A 41CB052A) & (W179-9) & (A41CB052A) & (VV-179-4) \\
\hline PAK 41 & G1-2 & $\mathrm{P}[8]-3$ & G1-2 & G1-3 & $\mathrm{P}[8]-1$ & $P[8]-2$ & 96.7 & 91.2 & 87.6 & 86.9 \\
\hline PAK 65 & G1-2 & $P[8]-3$ & G1-2 & G1-3 & $\mathrm{P}[8]-1$ & $P[8]-2$ & 96.7 & 91.2 & 87.6 & 86.9 \\
\hline PAK 77 & G1-2 & $\mathrm{P}[8]-3$ & G1-2 & G1-3 & $\mathbf{P}[8]-1$ & $P[8]-2$ & 96.7 & 91.2 & 87.6 & 86.9 \\
\hline PAK 88 & G1-1 & $\mathrm{p}[8]-4$ & G1-2 & G1-3 & $\mathrm{P}[8]-1$ & $\mathrm{P}[8]-2$ & 93.2 & 90.5 & 88.8 & 91.7 \\
\hline PAK540 & G1-1 & $\mathrm{p}[8]-4$ & $\mathrm{G} 1-2$ & G1-3 & $\mathrm{P}[8]-1$ & $\mathrm{p}[8]-2$ & 93.2 & 90.5 & 88.8 & 91.7 \\
\hline PAK601 & G1-1 & $\mathrm{P}[8]-4$ & G1-2 & G1-3 & $\mathrm{P}[8]-1$ & $\mathrm{P}[8]-2$ & 93.2 & 90.5 & 88.8 & 91.7 \\
\hline \multicolumn{11}{|c|}{ Amino acid identities (\%) } \\
\hline PAK41 & G1-2 & $P[8]-3$ & G1-2 & G1-3 & $P[8]-1$ & $\mathrm{P}[8]-2$ & 96.3 & 93.5 & 92.3 & 92.1 \\
\hline PAK 65 & G1-2 & $\mathrm{P}[8]-3$ & G1-2 & G1-3 & $P[8]-1$ & $\mathrm{P}[8]-2$ & 96.3 & 93.5 & 92.3 & 92.1 \\
\hline PAK 77 & G1-2 & $P[8]-3$ & G1-2 & G1-3 & $\mathrm{P}[8]-1$ & $\mathrm{P}[8]-2$ & 96.3 & 93.5 & 92.3 & 92.1 \\
\hline PAK 88 & G1-1 & $\mathrm{P}[8]-4$ & G1-2 & G1-3 & $\mathrm{P}[8]-1$ & $\mathrm{P}[8]-2$ & 94.2 & 93.2 & 93.6 & 95.4 \\
\hline PAK540 & G1-1 & $\mathrm{P}[8]-4$ & G1-2 & G1-3 & $P[8]-1$ & $\mathrm{P}[8]-2$ & 94.2 & 93.2 & 93.6 & 95.4 \\
\hline PAK601 & G1-1 & $\mathrm{P}[8]-4$ & G1-2 & G1-3 & $\mathrm{P}[8]-1$ & $\mathrm{P}[8]-2$ & 94.2 & 93.2 & 93.6 & 95.4 \\
\hline
\end{tabular}

FIGURE 1 | The nucleotides and amino acids differences of VP7 and VP4 proteins of Pakistani G1P[8] strains with rotavirus vaccine strains. The similarities between G1 lineage I to Rotarix ${ }^{\mathrm{TM}}$ and RotaTeq ${ }^{\mathrm{TM}}$ vaccine strains is shown in green and purple colours, respectively. While, the similarities between $\mathrm{G} 1$ lineage $/ I$ to Rotarix ${ }^{\mathrm{TM}}$ and RotaTeq ${ }^{\mathrm{TM}}$ vaccine strains is shown in blue and orange colours, respectively.

observed in two regions (7-1a and 7-1b). Comparing G1-lineage I strains with Rotarix ${ }^{\mathrm{TM}} \mathrm{G} 1 \mathrm{P}[8]$ strain, amino acid changes were observed at 2 positions in region 7-1a and at a single position in region 7-2. When G1-lineage I strains were compared with RotaTeq $^{\text {TM }}$ G1P[5] vaccine strain, amino acid changes at 3 and 2 positions were observed in region 7-1a and 7-2, respectively. Comparing G1-lineage II strains with the vaccine strain Rotarix $^{\mathrm{TM}} \mathrm{G} 1 \mathrm{P}[8]$, no single change in amino acid was observed. While, on comparison with RotaTeq $^{\mathrm{TM}}$ G1P[5] vaccine strain, two amino acid changes were observed, 1 in region 7-1a and 1in region 7-2 (Figure 4).

VP4 protein contains a total of 776 amino acids and is activated by the proteolytic cleavage into two subunits, i.e., $\mathrm{VP}^{*}$ and VP5*. The VP8* subunit consists of four antigenic regions, i.e., 8-1-8-4, while the VP5* subunit contains five antigenic regions, i.e., 5-1-5-5, that overall contain 37 amino acids (41). Comparing VP8* antigenic epitopes with RotaTeq ${ }^{\text {TM }}$ and Rotarix ${ }^{\mathrm{TM}}$ vaccines amino acid differences were observed in three regions with the exception of region 8-4 which was found to be conserved in both our study and vaccine strains. When comparing VP5* antigenic epitopes with RotaTeq ${ }^{\mathrm{TM}}$ and Rotarix ${ }^{\mathrm{TM}}$ vaccines, amino acid changes were observed in one region 5-1, while four regions were found to be conserved for both our study and vaccine strains.

When our study $\mathrm{P}[8]-3$ strains were compared with Rotarix ${ }^{\mathrm{TM}}$ G1P[8] and RotaTeq ${ }^{\text {TM }}$ G6P[8] vaccine strains, amino acids changes were observed at 14 and 12 positions, respectively. For Rotarix ${ }^{\mathrm{TM}}$, changes were observed at positions $8,2,3$, and 1 in regions 8-1, 8-2, and 8-3 and 5-1, respectively. For RotaTeq ${ }^{\mathrm{TM}}$, changes were observed at positions 8,2 , and 2 in regions $8-1$ and 8-2 and 5-1, respectively. When our study $\mathrm{P}[8]-4$ strains were compared with Rotarix ${ }^{\mathrm{TM}} \mathrm{G} 1 \mathrm{P}[8]$ and RotaTeq ${ }^{\mathrm{TM}} \mathrm{G} 6 \mathrm{P}[8]$ vaccine strains, amino acid substitutions were observed at 14 and 12 positions, respectively. For Rotarix ${ }^{\mathrm{TM}}$, in regions $8-1,8-2,8-3$, and 5-1 amino acid changes were observed at $8,2,2$, and 2 positions, respectively. For RotaTeq ${ }^{\mathrm{TM}}$, in regions $8-1,8-2,8-3$, and 5-1 amino acid changes were observed at $8,2,1$, and 1 positions, respectively. Overall, most of the changes detected in the Pakistani RVA viruses were identified in the VP4 head part $\left(\mathrm{VP}^{\star}\right)$ and very few changes were observed in the body of the spike or $\mathrm{VP}^{\star}$ (Figure 5).

\section{DISCUSSION}

Rotavirus gastroenteritis accounts for significant mortality rates in children under 5 years of age (42). The World Health Organization (WHO) has prioritised the development and introduction of vaccines to manage this disease due to the large global burden of rotavirus (43). Two Rotarix ${ }^{\mathrm{TM}}$ and RotaTeq $^{\text {TM }}$ RV vaccines recently implemented in children's immunisation programs in many countries around the world are highly successful in helping to prevent RV infections (23). Both vaccines have been shown to be successful against commonly circulating genotypes of RVs including G1-4, G9, and $\mathrm{P}[4], \mathrm{P}[6]$, and $\mathrm{P}[8]$ (25). However, as a result of the longterm use of such vaccines, possible vaccine escape strains could be selected in the future $(44,45)$.

In the current study, we compared antigenic differences between VP7-G1 and VP4-P8 (VP8 $\left.{ }^{*}, \mathrm{VP}^{*}\right)$ proteins of circulating human RVA strains in Pakistan and two available vaccines (RotaTeq ${ }^{\mathrm{TM}}$ and Rotarix ${ }^{\mathrm{TM}}$ ). Of the two lineages (lineage I and II) of VP7-G1 strains circulating in Pakistan, lineage I showed a few amino acids variations from both RotaTeq ${ }^{\text {TM }}$ and Rotarix $^{\mathrm{TM}}$ belong to lineages II and III, respectively. Pakistani G1-lineage II strains, however, showed remarkable similarities to the Rotarix ${ }^{\mathrm{TM}}$ vaccine strain.

RV glycosylation increases their pathogenicity and enhances their resistance to mAbs (46). Two N-linked glycosylation sites at positions 69 and 238 have been identified in the G1 genotype of 


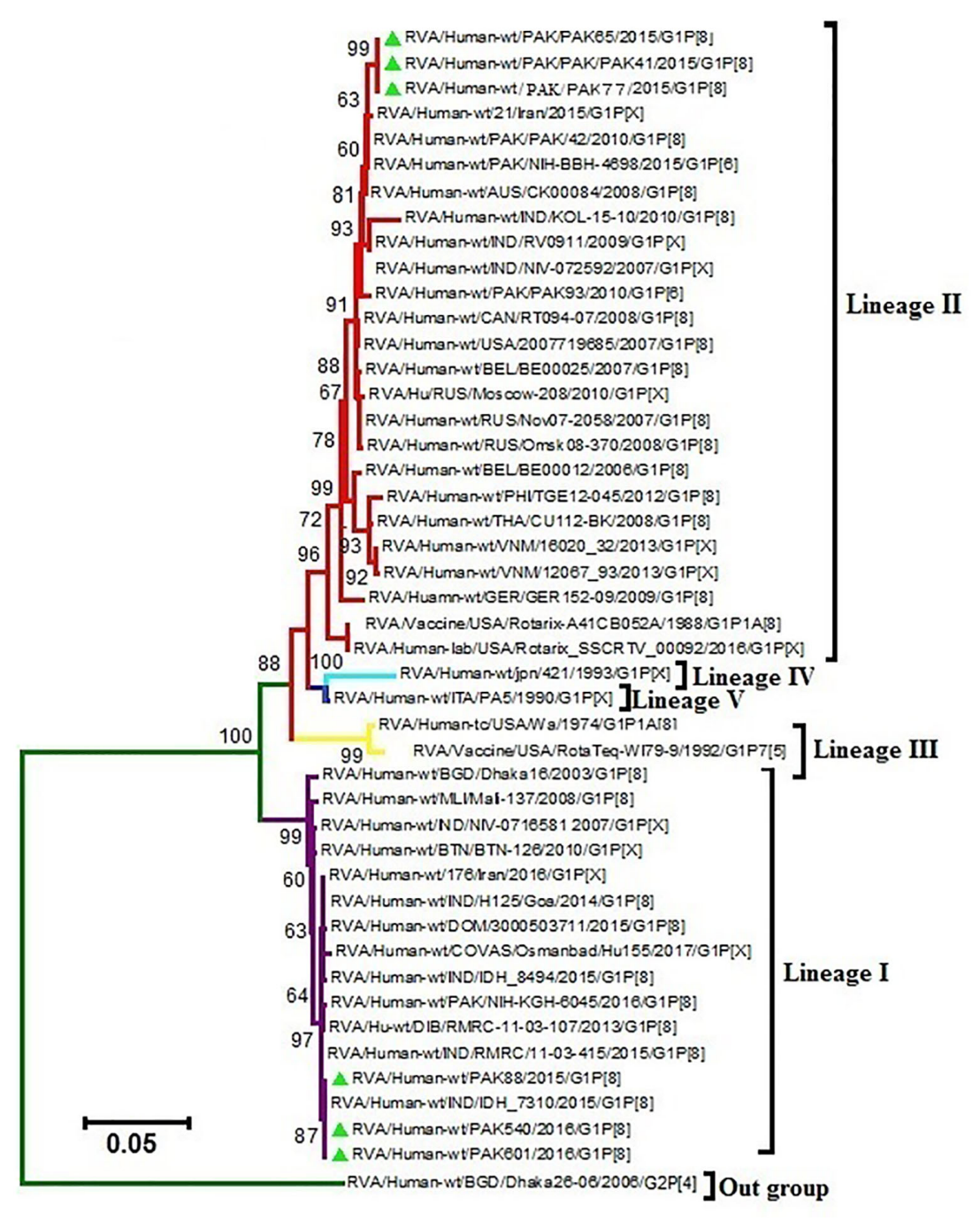

FIGURE 2 | Maximum Likelihood tree of the VP7 protein of G1P[8] group A rotavirus (RVA) strains reported in Pakistan and the vaccine strains Rotarix ${ }^{\mathrm{TM}}$ and RotaTeq $^{\mathrm{TM}}$. Maximum Likelihood trees were generated using Kimura-2-parameter model in MEGA 6. Coloured branches represent different lineages. The lineage I is represented by purple colour branch including strains isolated from all over the world and our study strains represented by green triangles in the same branch. The lineage II is represented by red colour branch including strains isolated from all over the world and our study strains represented by green triangle in the same branch. Yellow colour branch includes strains belong to lineage III. Aqua colour branch includes strains belong to lineage IV. Blue colour branch includes strains belong to lineage V. G2P[4] RVA strain from Bangladesh is used as an out-group represented by green colour branch. Bootstrap replicates (1000) below than 60\% are not shown in the tree.

VP7 protein vaccine strains, which were found to be conserved in the Pakistani RVA G1P[8] strains. Amino acid substitution S123N, N94S, and M217 T have been observed between strains of G1- lineage I. The rotavirus vaccine was introduced in Pakistan in 2018. It was initially proposed that the strains found in 2015-2016 may occur in our natural population. However, these variations were also reported between the G1P [8] strain in Australia, Belgium, Labenon, Russia and India (25, $33,47-49)$. It was suggested, therefore, that they could have been introduced into Pakistan from other countries of the world.

In this study, strains of G1 lineage I are paired with lineage IV of $\mathrm{P}[8]$ and G1 lineage II with $\mathrm{P}[8]$ lineage III. In the previous studies reported in Belgium and India, lineage I strains are coupled with lineage III of P[8] and lineage II of G1 paired with lineage IV of $\mathrm{P}[8](33,47)$. Thus, a full genome analysis of these strains is proposed in the future that may better clarify these genotypic interchanges between the different G1P [8] strains.

Our study's P[8] lineage III and IV genotypes are quite different from the lineages I and II, respectively, of both Rotarix $^{\mathrm{TM}}$ and RotaTeq ${ }^{\mathrm{TM}}$. The lineage IV strains are also called OP354-like or P[8]-b. These OP354-like strains have recently gained interest because these are being detected all over the world after being first reported in Malawi (50). These findings are supported by phylogenetic analysis, where lineage III and IV strains clustered away from vaccine strains and showed 


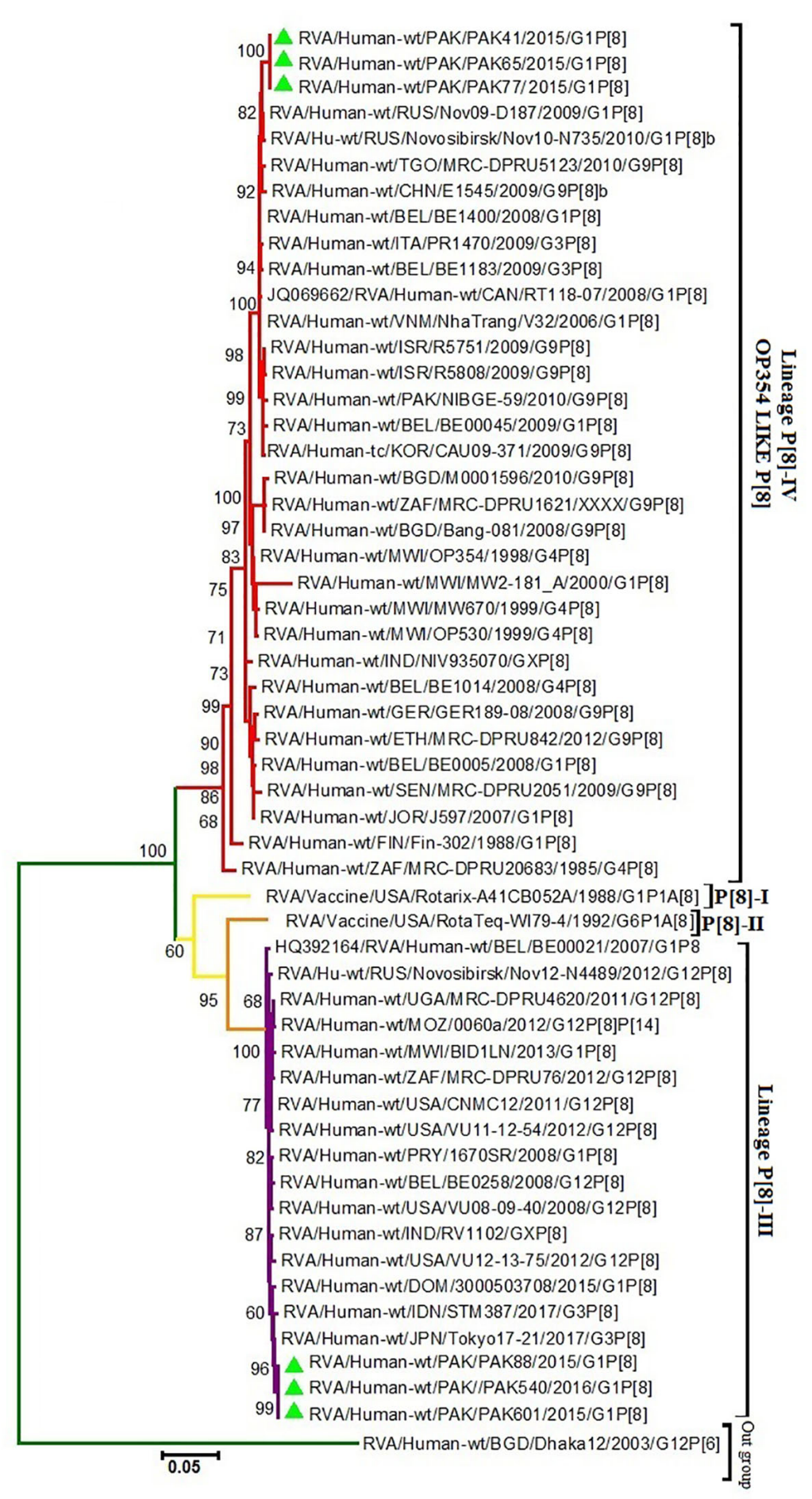

FIGURE 3 | Maximum Likelihood Phylogenetic tree of the VP4 protein of G1P[8] Group A rotavirus (RVA) strains reported in Pakistan and the vaccine strains Rotarix ${ }^{\mathrm{TM}}$ and RotaTeq ${ }^{\mathrm{TM}}$. Maximum Likelihood trees were generated using Kimura-2-parameter model in MEGA 6. Coloured branches represent different lineages. Yellow colour branch includes strains belong to lineage I and orange colour branch represent lineage II strains. The lineage III is represented by Purple colour branch including strains isolated from all over the world and our study strains represented by green triangles in the same branch. The lineage IV is represented by Red colour branch including strains isolated from all over the world and our study strains represented by green triangle in the same branch. RVA strain (G12P[6]) from Bangladesh is used as an as out-group is represented by green colour branch in the tree. Bootstrap replicates (1000) below than 60\% are not shown in the tree. 


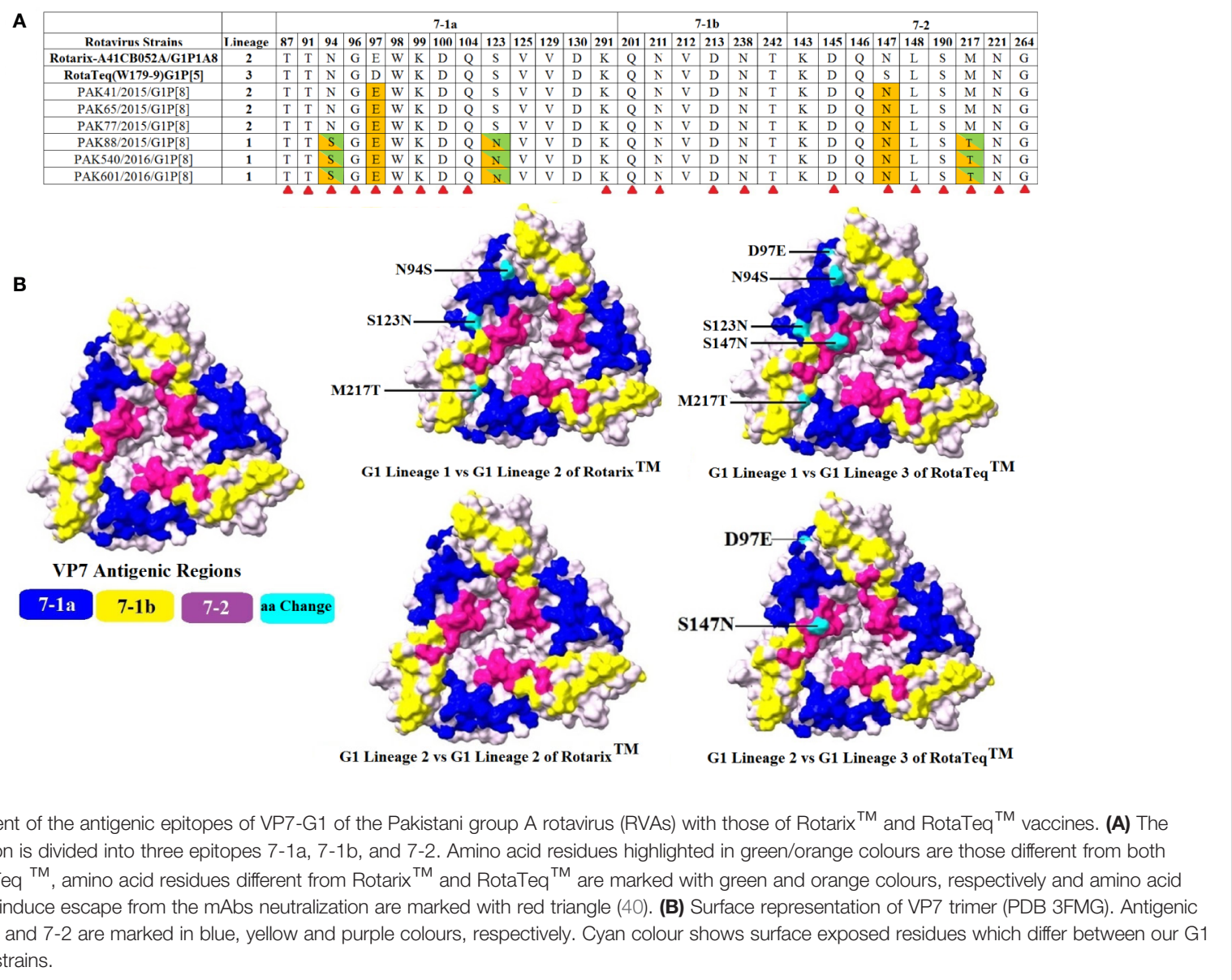

FIGURE 4 | Alignment of the antigenic epitopes of VP7-G1 of the Pakistani group A rotavirus (RVAs) with those of Rotarix ${ }^{\text {TM }}$ and RotaTeq ${ }^{\text {TM }}$ vaccines. (A) The whole antigenic region is divided into three epitopes 7-1a, 7-1b, and 7-2. Amino acid residues highlighted in green/orange colours are those different from both Rotarix $^{\mathrm{TM}}$ and RotaTeq ${ }^{\mathrm{TM}}$, amino acid residues different from Rotarix ${ }^{\mathrm{TM}}$ and RotaTeq ${ }^{\mathrm{TM}}$ are marked with green and orange colours, respectively and amino acid residues believed to induce escape from the mAbs neutralization are marked with red triangle (40). (B) Surface representation of VP7 trimer (PDB 3FMG). Antigenic epitopes 7-1a, 7-1b, and 7-2 are marked in blue, yellow and purple colours, respectively. Cyan colour shows surface exposed residues which differ between our G1 strains and vaccine strains.

close relationships with previously reported RVA strains from Japan and Russia, respectively.

Significant variations in the genes of VP7 may impair immunity, resulting in ineffective results or vaccine response failure. In contrast with the $\mathrm{G}$ types, poor immune responses to $\mathrm{P}$ [8] strains can be compensated for due to the relative lack of diversity between P-types (25). It has been shown that the immune response to the VP4 antigen is important and there are cross-reactive epitopes on the VP4 protein. Compared with the $G$ types, the relative lack of diversity among P-types may contribute to heterotypic immune response (51).

Studies from Europe and America showed that variations in the sequence of VP7 and VP4 genes of circulating RVA strains and vaccine strains in these countries had no effect on the efficacy of the vaccines (47). In fact, Rotarix ${ }^{\mathrm{TM}}$ a monovalent rotavirus vaccine is believed to be effective against non G1P[8] rotavirus strains (52). The conserved immunogenic regions in VP7 and VP4 genotypes and non-structural proteins or internal proteins seems to be the reason for this cross protection (18). Earlier studies have shown that primary rotavirus infections are associated with rises in serum antibody levels to the structural proteins VP2, VP4, VP6, VP7, and to the non-structural proteins NSP2 and NSP4 $(53,54)$.
Since both vaccines have shown considerable efficacy to date in Europe and America, however, their long-term use has been suggested to contribute to the selection of strains capable of avoiding vaccine-induced immunity (44). However, after the introduction of Rotarix ${ }^{\mathrm{TM}}$ in Belgium, the incidence of Rotarixlike G1P[8] strains has declined more than the G1P[8] strains belonging to $\mathrm{G} 1$ and $\mathrm{P}[8]$ lineages that are less similar to $\operatorname{Rotarix}^{\mathrm{TM}}$ (33).

Overall, the results of the current study showed potential antigenic disparities in circulating G1P[8] strains prior to the RV vaccines introduction in Pakistan compared to Rotarix ${ }^{\mathrm{TM}}$ and RotaTeq $^{\mathrm{TM}}$ vaccines. The findings of this study will provide baseline information for a post-vaccine study to gain insight into the effect of the introduction of the vaccines on the diversity of the rotavirus genotypes in Pakistan with respect to their subgenotypic lineages. But the exact effect of differences in amino acids in antigenic epitopes cannot be predicted from sequence and structural information alone. We believe that the findings of this research will be strengthened by continuous surveillance of circulating RVAs in line with increasing sample size and vaccine matching evaluation using genotype-specific antibodies and neutralization assays. 


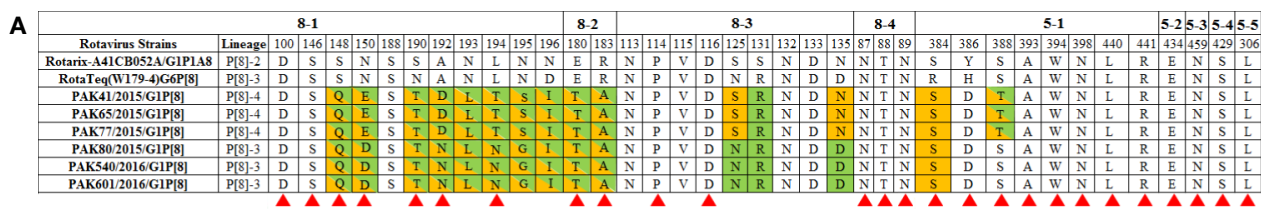

B
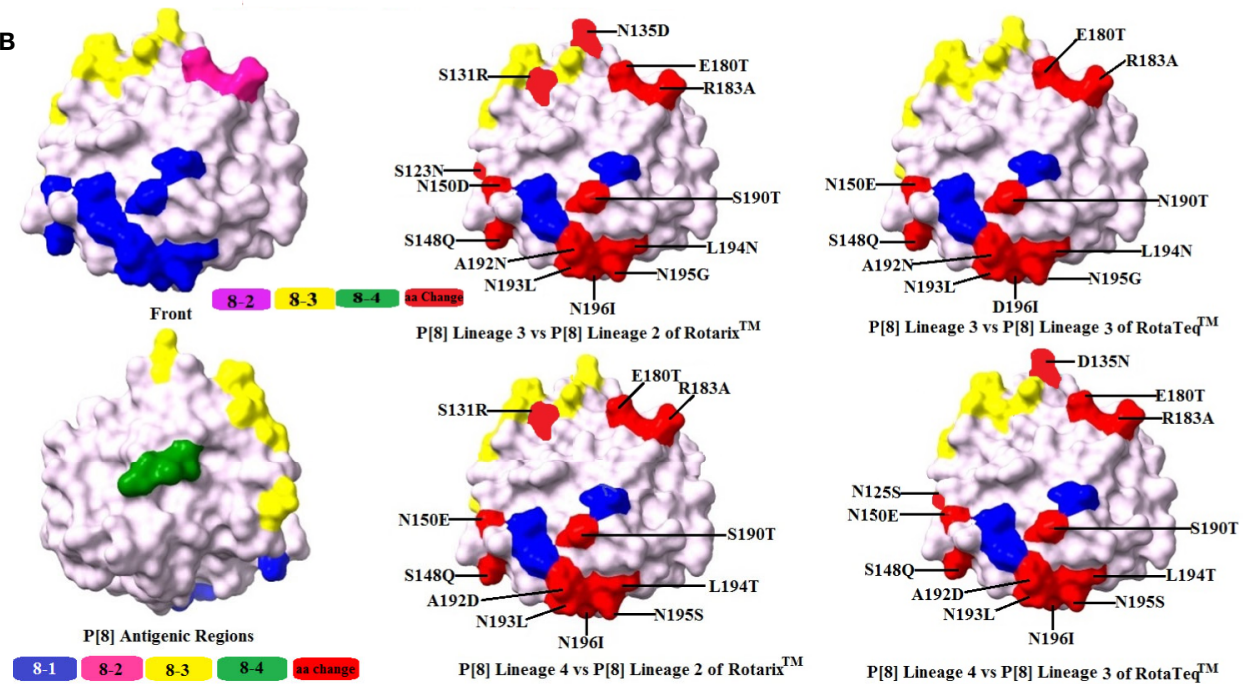

$P[8]$ Lineage 4 vs P[8] Lineage 2 of Rotarix ${ }^{\text {TM }}$

$\mathrm{P}[8]$ Lineage 4 vs $\mathrm{P}[8]$ Lineage 3 of RotaTeq ${ }^{\mathrm{MM}}$

FIGURE 5 | Alignment of the antigenic epitopes of VP4-P[8] of the Pakistani group A rotavirus (RVAs) with those of Rotarix ${ }^{\mathrm{TM}}$ and RotaTeq ${ }^{\mathrm{TM}}$ vaccines. (A) The whole antigenic region is divided into four epitopes 8-1, 8-2, 8-3, and 8-4 for VP8* and five epitopes for VP5*. Amino acid residues shading in green and orange are those different from Rotari ${ }^{\mathrm{TM}}$ and RotaTeq ${ }^{\mathrm{TM}}$, respectively, amino acid residues highlighting in green/orange colour are those different from both Rotarix ${ }^{\mathrm{TM}}$ and RotaTeq $^{\text {TM }}$ and amino acid residues believed to induce escape from the mAbs neutralization are marked with red triangle (41). (B) Representation of surface exposed amino acid residues of VP4 core VP8* core (PDB 1KQR). Antigenic epitopes 8-1, 8-2, 8-3, and 8-4 are represented by blue, pink, yellow, and green colours, respectively. Red colour shows surface exposed residues which differ between our P[8] strains and vaccine strains.

\section{DATA AVAILABILITY STATEMENT}

The datasets presented in this study can be found in online repositories. The names of the repository/repositories and accession number(s) can be found in the article.

\section{ETHICS STATEMENT}

The studies involving human participants were reviewed and approved by Ethical Committee of PIMS and Benazir Bhutto Hospitals and Internal Review Board of COMSATS University, Islamabad, Pakistan. Written informed consent to participate in this study was provided by the participants' legal guardian/next of kin.

\section{REFERENCES}

1. Troeger C, Khalil IA, Rao PC, Cao S, Blacker BF, Ahmed T, et al. Rotavirus Vaccination and the Global Burden of Rotavirus Diarrhea among Children Younger Than 5 Years. JAMA Pediatr (2018) 172:958-65. doi: 10.1001/ jamapediatrics.2018.1960

2. Schwartz LM, Zaman K, Basunia AH, Syed A, Faruque G, Ahmed T, et al. Impact of Rotavirus Vaccine Introduction in Children Less Than 2 Years of

\section{AUTHOR CONTRIBUTIONS}

AS (COMSATS University, Islamabad, Pakistan) planned the project and carried out experiments, data analysis and composed article. NB (COMSATS University, Pakistan) created idea and helped to start writing the article. All authors contributed to the article and approved the submitted version.

\section{FUNDING}

This study was funded by Pakistan's Higher Education Commission (HEC), Grant number (2BM1-196) as part of a Doctorate programme.
Age Presenting for Medical Care With Diarrhea in Rural Matlab, Bangladesh. Clin Infect Dis (2019) 69:2059-70. doi: 10.1093/cid/ciz133

3. Tate JE, Burton AH, Boschi-Pinto C, Parashar UDWorld Health Organization-Coordinated Global Rotavirus Surveillance Network for the WHOGRS, , Agocs M, et al. Global, Regional, and National Estimates of Rotavirus Mortality in Children $<5$ Years of Age, 2000-2013. Clin Infect Dis (2016) 62(Suppl 2):S96-S105. doi: 10.1093/cid/civ1013

4. WHO. Estimated rotavirus deaths for children under 5 years of age: 2013, 215 000. WHO (2016). Available at: https://www.who.int/immunization/ 
monitoring_surveillance/burden/estimates/rotavirus/en/ (Accessed October 24, 2018).

5. Sadiq A, Bostan N, Yinda KC, Naseem S, Sattar S. Rotavirus: Genetics, pathogenesis and vaccine advances. Rev Med Virol (2018) 28:e2003. doi: $10.1002 /$ rmv.2003

6. Kaneko M, Takanashi S, Thongprachum A, Hanaoka N, Fujimoto T, Nagasawa $K$, et al. Identification of vaccine-derived rotavirus strains in children with acute gastroenteritis in Japan, 2012-2015. PLos One (2017) 12:e0184067. doi: 10.1371/journal.pone.0184067

7. Sadiq A, Bostan N, Bokhari H, Matthijnssens J, Yinda KC, Raza S, et al. Molecular characterization of human group A rotavirus genotypes circulating in Rawalpindi, Islamabad, Pakistan during 2015-2016. PLoS One (2019) 14: e0220387. doi: 10.1371/journal.pone.0220387

8. Heylen E, Zeller M, Ciarlet M, Lawrence J, Steele D, Van Ranst M, et al. Comparative analysis of pentavalent rotavirus vaccine strains and G8 rotaviruses identified during vaccine trial in Africa. Sci Rep (2015) 5:14658. doi: $10.1038 /$ srep 14658

9. RCWG. Rotavirus Classification Working Group: RCWG - Laboratory of Viral Metagenomics (2018). Available at: https://rega.kuleuven.be/cev/ viralmetagenomics/virus-classification/rcwg (Accessed June 7, 2019).

10. Gutierrez MB, Fialho AM, Maranhão AG, Malta FC, de Andrade J da SR, de Assis RMS, et al. Rotavirus A in Brazil: Molecular epidemiology and surveillance during 2018-2019. Pathogens (2020) 9:1-15. doi: 10.3390/ pathogens 9070515

11. Ogden KM, Tan Y, Akopov A, Stewart LS, McHenry R, Fonnesbeck CJ, et al. Multiple Introductions and Antigenic Mismatch with Vaccines May Contribute to Increased Predominance of G12P[8] Rotaviruses in the United States Our findings emphasize the need for continued monitoring of circulating rotaviruses Downloaded from. J Virol (2019) 93:1476-94. doi: 10.1128/JVI

12. Kirkwood CD. Genetic and Antigenic Diversity of Human Rotaviruses: Potential Impact on Vaccination Programs. J Infect Dis (2010) 202:S43-8. doi: $10.1086 / 653548$

13. Jere KC, Chaguza C, Bar-Zeev N, Lowe J, Peno C, Kumwenda B, et al. Emergence of Double- and Triple-Gene Reassortant G1P[8] Rotaviruses Possessing a DS-1-Like Backbone after Rotavirus Vaccine Introduction in Malawi. J Virol (2017) 92. doi: 10.1128/JVI.01246-17

14. Agbla JMM, Capo-Chichi A, Agbankpé AJ, Dougnon TV, Yadouleton AWM, Houngbégnon O, et al. Epidemiological Survey of Rotaviruses Responsible for Infantile Diarrhea by the Immunomolecular Technique in Cotonou (Benin, West Africa). Int J Microbiol (2018) 2018:3602967. doi: 10.1155/2018/3602967

15. Isanaka S, Guindo O, Langendorf C, Matar Seck A, Plikaytis BD, SayinzogaMakombe N, et al. Efficacy of a Low-Cost, Heat-Stable Oral Rotavirus Vaccine in Niger. N Engl J Med (2017) 376:1121-30. doi: 10.1056/NEJMoa1609462

16. Lestari FB, Vongpunsawad S, Wanlapakorn N, Poovorawan Y. Rotavirus infection in children in Southeast Asia 2008-2018: Disease burden, genotype distribution, seasonality, and vaccination. J BioMed Sci (2020) 27:1-19. doi: 10.1186/s12929-020-00649-8

17. Bhandari N, Rongsen-Chandola T, Bavdekar A, John J, Antony K, Taneja S, et al. Efficacy of a monovalent human-bovine (116E) rotavirus vaccine in Indian infants: A randomised, double-blind, placebo-controlled trial. Lancet (2014) 383:2136-43. doi: 10.1016/S0140-6736(13)62630-6

18. Ward RL, Clark HF, Offit PA. Influence of Potential Protective Mechanisms on the Development of Live Rotavirus Vaccines. J Infect Dis (2010) 202:S72-9. doi: $10.1086 / 653549$

19. Abou-Nader AJ, Sauer MA, Steele AD, Tate JE, Atherly D, Parashar UD, et al. Global rotavirus vaccine introductions and coverage: 2006-2016. Hum Vaccines Immunother (2018) 14:2281-96. doi: 10.1080/21645515.2018.1470725

20. Rota Council. Global Introduction Status | Rota Council (2020). Available at: $\mathrm{http}: / /$ rotacouncil.org/vaccine-introduction/global-introduction-status/ (Accessed August 5, 2019).

21. Nelson EAS, Glass RI. Rotavirus: Realising the potential of a promising vaccine. Lancet (2010) 376:568-70. doi: 10.1016/S0140-6736(10)60896-3

22. Palombo EA. Genetic and antigenic diversity of human rotaviruses: potential impact on the success of candidate vaccines. FEMS Microbiol Lett (1999) 181:1-8. doi: 10.1111/j.1574-6968.1999.tb08819.x

23. Burke RM, Tate JE, Kirkwood CD, Steele AD, Parashar UD. Current and new rotavirus vaccines. Curr Opin Infect Dis (2019) 32:435-44. doi: 10.1097/ QCO.0000000000000572
24. Deen J, Lopez AL, Kanungo S, Wang XY, Anh DD, Tapia M, et al. Improving rotavirus vaccine coverage: Can newer-generation and locally produced vaccines help? Hum Vaccines Immunother (2018) 14:495-9. doi: 10.1080/ 21645515.2017.1403705

25. Harastani HH, Reslan L, Sabra A, Ali Z, Hammadi M, Ghanem S, et al. Genetic Diversity of Human Rotavirus A Among Hospitalized Children Under-5 Years in Lebanon. Front Immunol (2020) 11:317. doi: 10.3389/ fimmu.2020.00317

26. Read AF, Baigent SJ, Powers C, Kgosana LB, Blackwell L, Smith LP, et al. Imperfect Vaccination Can Enhance the Transmission of Highly Virulent Pathogens. PLoS Biol (2015) 13:e1002198. doi: 10.1371/journal.pbio.1002198

27. Posteraro B, Pastorino R, Di Giannantonio P, Ianuale C, Amore R, Ricciardi $\mathrm{W}$, et al. The link between genetic variation and variability in vaccine responses: Systematic review and meta-analyses. Vaccine (2014) 32:1661-9. doi: 10.1016/j.vaccine.2014.01.057

28. Arista S, Giammanco GM, De Grazia S, Ramirez S, Lo Biundo C, Colomba C, et al. Heterogeneity and Temporal Dynamics of Evolution of G1 Human Rotaviruses in a Settled Population. J Virol (2006) 80:10724-33. doi: 10.1128/ jvi.00340-06

29. Umair M, Abbasi BH, Nisar N, Alam MM, Sharif S, Shaukat S, et al. Molecular analysis of group A rotaviruses detected in hospitalized children from Rawalpindi, Pakistan during 2014. Infect Genet Evol (2017) 53:160-6. doi: 10.1016/j.meegid.2017.05.009

30. Tamim S, Hasan F, Matthijnssens J, Sharif S, Shaukat S, Alam MM, et al. Epidemiology and phylogenetic analysis of VP7 and VP4 genes of rotaviruses circulating in Rawalpindi, Pakistan during 2010. Infect Genet Evol (2013) 14:161-8. doi: 10.1016/j.meegid.2012.10.009

31. Kazi AM, Warraich GJ, Qureshi S, Qureshi H, Khan MMA, Zaidi AKM. Group and members of the PRS. Sentinel Hospital-Based Surveillance for Assessment of Burden of Rotavirus Gastroenteritis in Children in Pakistan. PLoS One (2014) 9:e108221. doi: 10.1371/journal.pone.0108221

32. Magagula NB, Esona MD, Nyaga MM, Stucker KM, Halpin RA, Stockwell TB, et al. Whole genome analyses of G1P [8] rotavirus strains from vaccinated and non-vaccinated South African children presenting with diarrhea. J Med Virol (2015) 87:79-101. doi: 10.1002/jmv.23971

33. Zeller M, Patton JT, Heylen E, De Coster S, Ciarlet M, Van Ranst M, et al. Genetic Analyses Reveal Differences in the VP7 and VP4 Antigenic Epitopes between Human Rotaviruses Circulating in Belgium and Rotaviruses in Rotarix and RotaTeq. J Clin Microbiol (2012) 50:966-76. doi: 10.1128/ JCM.05590-11

34. Gouvea V, Glass RI, Woods P, Taniguchi K, Clark HF, Forrester B, et al. Polymerase chain reaction amplification and typing of rotavirus nucleic acid from stool specimens. J Clin Microbiol (1990) 28:276-82. doi: 10.1128/ JCM.28.2.276-282.1990

35. Nagashima S, Kobayashi N, Paul SK, Alam MM, Chawla-Sarkar M, Krishnan T. Characterization of full-length VP4 genes of OP354-like P[8] human rotavirus strains detected in Bangladesh representing a novel P[8] subtype. Arch Virol (2009) 154:1223-31. doi: 10.1007/s00705-009-0436-3

36. Matthijnssens J, Rahman M, Martella V, Xuelei Y, De Vos S, De Leener K, et al. Ranst M Van. Full Genomic Analysis of Human Rotavirus Strain B4106 and Lapine Rotavirus Strain 30/96 Provides Evidence for Interspecies Transmission. J Virol (2006) 80:3801-10. doi: 10.1128/JVI.80.8.38013810.2006

37. Tamura K, Stecher G, Peterson D, Filipski A, Kumar S. MEGA6: Molecular Evolutionary Genetics Analysis Version 6.0. Mol Biol Evol (2013) 30:2725-9. doi: $10.1093 / \mathrm{molbev} / \mathrm{mst} 197$

38. Pettersen EF, Goddard TD, Huang CC, Couch GS, Greenblatt DM, Meng EC, et al. UCSF Chimera - A visualization system for exploratory research and analysis. J Comput Chem (2004) 25:1605-12. doi: 10.1002/jcc.20084

39. Dormitzer PR, Greenberg HB, Harrison SC. Purified recombinant rotavirus VP7 forms soluble, calcium-dependent trimers. Virology (2000) 277:420-8. doi: 10.1006/viro.2000.0625

40. Aoki ST, Settembre EC, Trask SD, Greenberg HB, Harrison SC, Dormitzer PR. Structure of rotavirus outer-layer protein VP7 bound with a neutralizing Fab. Sci (80-) (2009) 324:1444-7. doi: 10.1126/science.1170481

41. Dormitzer PR, Nason EB, Prasad BVV, Harrison SC. Structural rearrangements in the membrane penetration protein of a non-enveloped virus. Nature (2004) 430:1053-8. doi: 10.1038/nature02836 
42. Aliabadi N, Antoni S, Mwenda JM, Weldegebriel G, Biey JNM, Cheikh D, et al. Global impact of rotavirus vaccine introduction on rotavirus hospitalisations among children under 5 years of age, 2008-16: findings from the Global Rotavirus Surveillance Network. Lancet Glob Heal (2019) 7:e893-903. doi: 10.1016/S2214-109X(19)30207-4

43. Jiang V, Jiang B, Tate J, Parashar UD, Patel MM. Performance of rotavirus vaccines in developed and developing countries. Hum Vaccin (2010) 6:53242. doi: 10.4161/hv.6.7.11278

44. Matthijnssens J, Bilcke J, Ciarlet M, Martella V, Bányai K, Rahman M, et al. Rotavirus disease and vaccination: impact on genotype diversity. Future Microbiol (2009) 4:1303-16. doi: 10.2217/fmb.09.96

45. Fujii Y, Hai Doan Y, Wahyuni RM, Lusida MI, Utsumi T, Shoji I, et al. Improvement of rotavirus genotyping method by using the semi-nested multiplex-PCR with new primer set. Front Microbiol (2019) 10:647. doi: $10.3389 /$ fmicb.2019.00647

46. Caust J, Dyall-Smith ML, Lazdins I, Holmes IH. Glycosylation, an important modifier of rotavirus antigenicity. Arch Virol (1987) 96:123-34. doi: 10.1007/ BF01320955

47. Kulkarni R, Arora R, Chitambar SD. Sequence analysis of VP7 and VP4 genes of G1P[8] rotaviruses circulating among diarrhoeic children in Pune, India: A comparison with Rotarix and RotaTeq vaccine strains. Vaccine (2014) 32: A75-83. doi: 10.1016/J.VACCINE.2014.03.080

48. Donato CM, Cowley D, Snelling TL, Akopov A, Kirkness EF, Kirkwood CD. Characterization of a G1P[8] rotavirus causing an outbreak of gastroenteritis in the Northern Territory, Australia, in the vaccine era. Emerg Microbes Infect (2014) 3:1-6. doi: 10.1038/emi.2014.47

49. Morozova OV, Sashina TA, Fomina SG, Novikova NA. Comparative characteristics of the VP7 and VP4 antigenic epitopes of the rotaviruses circulating in Russia (Nizhny Novgorod) and the Rotarix and RotaTeq vaccines. Arch Virol (2015) 160:1693-703. doi: 10.1007/s00705-015-2439-6
50. Damanka SA, Kwofie S, Dennis FE, Lartey BL, Agbemabiese CA, Doan YH, et al. Whole genome characterization and evolutionary analysis of OP354-like $\mathrm{P}$ [8] Rotavirus A strains isolated from Ghanaian children with diarrhoea. PLoS One (2019) 14:e0218348. doi: 10.1371/journal.pone.0218348

51. Steele DA, Neuzil KM, Cunliffe NA, Madhi SA, Bos P, Ngwira B, et al. Human rotavirus vaccine Rotarix ${ }^{\mathrm{TM}}$ provides protection against diverse circulating rotavirus strains in African infants: a randomized controlled trial. BMC Infectious Disease (2012) 12:213. doi: 10.1186/1471-2334-12-213

52. Vesikari T, Karvonen A, Prymula R, Schuster V, Tejedor J, Cohen R, et al. Efficacy of human rotavirus vaccine against rotavirus gastroenteritis during the first 2 years of life in European infants: randomised, double-blind controlled study. Lancet (2007) 370:1757-63. doi: 10.1016/S0140-6736(07)61744-9

53. Colomina J, Gil MT, Codoñer P, Buesa J. Viral proteins VP2, VP6, and NSP2 are strongly precipitated by serum and fecal antibodies from children with rotavirus symptomatic infection. J Med Virol (1998) 56:58-65. doi: 10.1002/ (SICI)1096-9071(199809)56:1<58::AID-JMV10>3.0.CO;2-S

54. Svensson L, Sheshberadaran H, Vene S, Norrby E, Grandien M, Wadell G. Serum antibody responses to individual viral polypeptides in human rotavirus infections. J Gen Virol (1987) 68:643-51. doi: 10.1099/0022-1317-68-3-643

Conflict of Interest: The authors declare that the research was conducted in the absence of any commercial or financial relationships that could be construed as a potential conflict of interest.

Copyright (c) 2020 Sadiq and Bostan. This is an open-access article distributed under the terms of the Creative Commons Attribution License (CC BY). The use, distribution or reproduction in other forums is permitted, provided the original author(s) and the copyright owner(s) are credited and that the original publication in this journal is cited, in accordance with accepted academic practice. No use, distribution or reproduction is permitted which does not comply with these terms. 